\title{
POLITICA FISCAL EN EE.UU. Y EUROPA: EFECTOS MACROECONOMICOS DE MEDIANO PLAZO*
}

\author{
FISCAL POLICY IN U.S. AND EUROPE: \\ MACROECONOMIC EFFECTS ON THE MEDIUM TERM
}

\section{ARI AISEN** \\ Fondo Monetario Internacional \\ DAVID MORENO*** \\ University of Maryland}

\begin{abstract}
Notwithstanding the effects of the expansive policies in the advanced economies, due to the 2008 financial crises, could raise their interest rates, generating a crowding-out effect over emerging market debt, this relation could not hold given the better fiscal position of these economies, such as Chile. In particular, higher interest rates in advanced economies could reflect a higher risk of default, consistent with their fiscal fragility.
\end{abstract}

Keywords: International finance, fiscal policy, emerging markets.

JEL Classification: F30, E62.

\section{Resumen}

Si bien el efecto de las políticas fiscales expansivas en las economías avanzadas, producto de la crisis financiera de 2008, podría elevar las tasas de interés en estos países, generando riesgos de un efecto desplazamiento de la deuda de países emergentes, esta relación no necesariamente permanecería frente a la solvencia fiscal relativa de algunos países emergentes como Chile. En particular, las mayores tasas de interés en países avanzados

\footnotetext{
* Se agradecen los comentarios de Rodrigo Cifuentes, Kevin Cowan, Eduardo Olaberría y Pablo Pincheira.

** E-mail: aaisen@imf.org.

*** Department of Economics, College Park, MD 20742, 3105 Tydings Hall. E-mail: moreno@econ.umd. edu.
} 
podrían reflejar mayor riesgo no pago, coherente con la fragilidad fiscal en dichos países.

Palabras Clave: Finanzas internacionales, política fiscal, mercados emergentes.

Clasificación JEL: F30, E62.

\section{INTRODUCCION}

La economía global fue fuertemente sacudida por la crisis financiera, que trajo asociada una fuerte pérdida de confianza por parte de los agentes económicos y la consecuente caída de la demanda agregada. Los gobiernos y bancos centrales salieron al rescate del sistema financiero introduciendo medidas tendientes a solucionar los problemas de liquidez y solvencia. Además, los bancos centrales redujeron las tasas de interés a niveles mínimos y los gobiernos adoptaron paquetes fiscales de magnitud expresiva (Gráfico 1), que incluyeron aumentos de gastos y recortes de impuestos no vistos en el período de la posguerra.

El objetivo principal de este esfuerzo combinado de política está centrado en un reavivamiento de la demanda agregada, a fin de recuperar el sendero de crecimiento del PIB. En el caso particular de la política fiscal, los paquetes estuvieron orientados a reforzar los estabilizadores automáticos tradicionales en períodos recesivos (especialmente en aquellos casos en que estos son menores), con medidas específicas enfocadas en maximizar el efecto multiplicador de las políticas. Además, estos paquetes contaron con una gama importante de medidas a fin de producir el efecto deseado sobre la demanda agregada (Gráfico 2).

Si bien es cierto que la expansión fiscal puede tener un efecto relevante sobre la demanda agregada en el corto y mediano plazo, los costos de largo plazo pueden ser significativos en términos del incremento de la deuda pública y el consecuente aumento de sus tasas de interés, que a su vez desplazan a la inversión y consumo privados. Más relevante para Chile, el aumento de las tasas de interés podría desplazar el financiamiento externo del país, afectando negativamente las condiciones de acceso de bancos y empresas residentes.

Este artículo tiene como objetivo analizar los efectos macroeconómicos de la expansión fiscal en curso en las economías desarrolladas, en especial en Estados Unidos y Reino Unido, países que registran algunos de los mayores déficits del mundo, en porcentaje del PIB (Gráfico 3). En este contexto, se busca responder dos preguntas cruciales: (1) ¿cuáles son los canales de transmisión por los cuales los déficits fiscales afectan a variables macroeconómicas y financieras en Estados Unidos y Reino Unido? y (2) ¿cómo los efectos de los déficits fiscales en estos países podrían transmitirse a países emergentes como Chile?

El artículo está estructurado como sigue: la Sección 2 presenta proyecciones del tamaño y composición del déficit y deuda fiscales en Estados Unidos y Europa; la Sección 3 discute las implicancias macroeconómicas de esta expansión fiscal con especial énfasis en los canales financieros que puedan afectar a Chile. La Sección 4 concluye. 


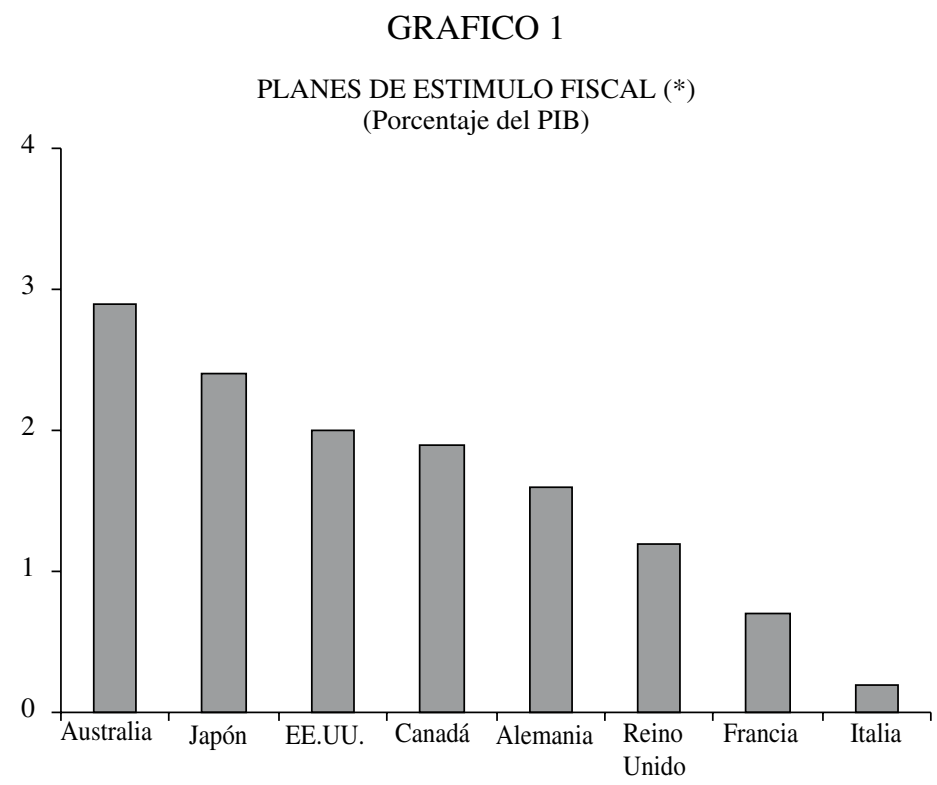

(*) Corresponde al gasto fiscal extraordinario comprometido para el año 2009 debido a la crisis financiera. Fuente: FMI (2009b).

\section{GRAFICO 2}

\section{ESTIMULO FISCAL DE ECONOMIAS AVANZADAS DEL G-20 POR CATEGORIA (2009-2010) (*)}

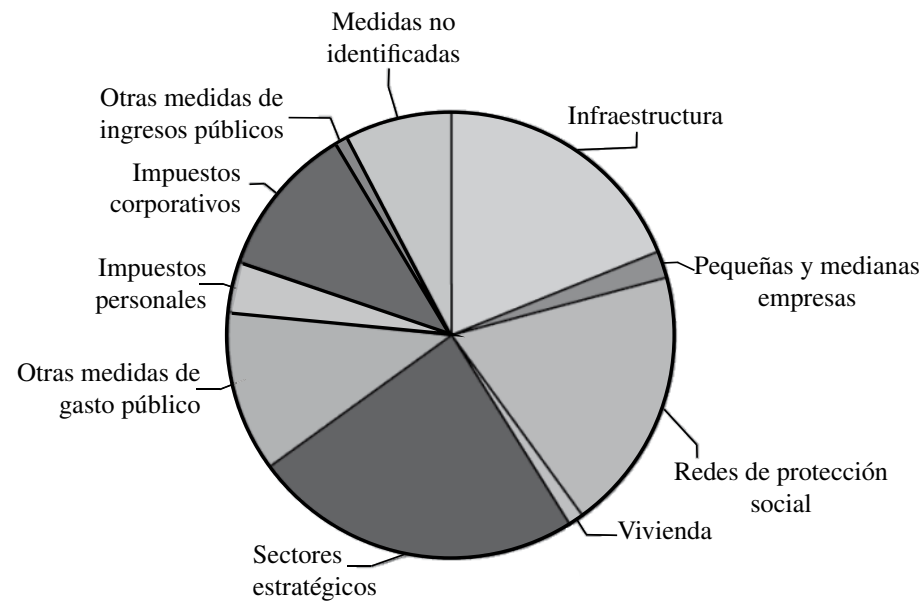

(*) No incluye medidas de apoyo al sector financiero.

Fuente: FMI (2009a). 

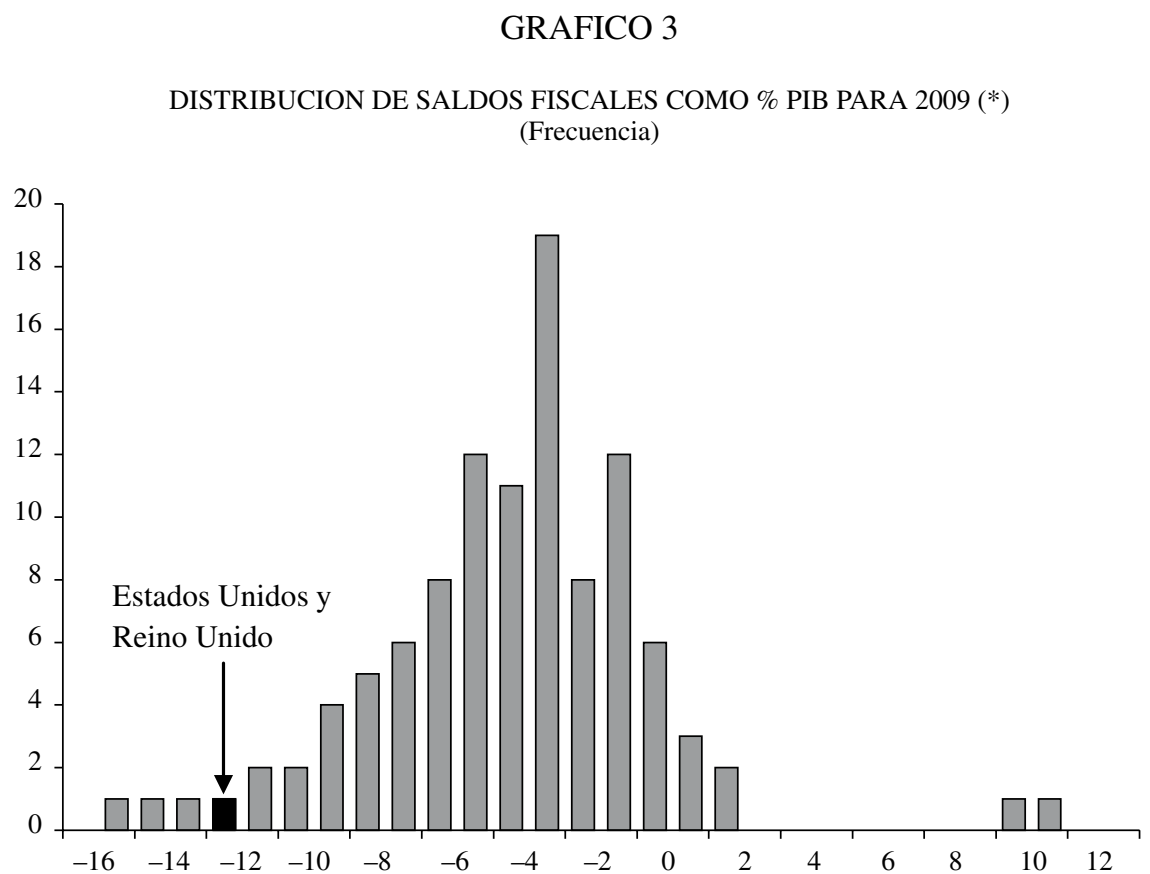

(*) Saldos fiscales financieros del gobierno general, como \% del PIB.

Fuente: Moody's Statistical Handbook, mayo de 2010.

\section{EVIDENCIA Y PROYECCIONES DE MEDIANO PLAZO}

Las políticas fiscales llevadas a cabo principalmente por las economías desarrolladas han buscado rescatar al sistema financiero y estimular la demanda agregada para tratar de reducir la duración y profundidad de la recesión económica. Estas políticas han llevado a estas economías a incurrir en enormes déficits fiscales, en muchos casos no vistos desde la Segunda Guerra Mundial, y una fuerte acumulación de la deuda pública.

En el caso de Estados Unidos y de Gran Bretaña, los costos directos netos de la ayuda al sistema financiero alcanzan a alrededor de 3,6\% y 5,4\% del PIB de 2009 (FMI, 2010), respectivamente, mientras los planes de estímulo fiscal no asociados al sector financiero ascienden a $2,0 \%$ y $1,4 \%$ en 2009 . Sumando a esto la importante caída en los ingresos fiscales, producto de la contracción del PIB, estos factores han llevado a estas economías a déficits que superan el 10\% del PIB en 2009. Fuentes oficiales como la Oficina Presupuestaria del Congreso (CBO, en inglés) en Estados Unidos y Her Majesty's Treasury (HM Treasury) en el Reino Unido estiman en sus últimos reportes que los déficits públicos llegarán a 2,6\% y 3,9\% hacia 2015, respectivamente; el FMI, por otra parte, estima que hacia el mismo período los déficits 


\section{GRAFICO 4}

\section{DEUDA PUBLICA (1) (2)}

(Porcentaje del PIB)
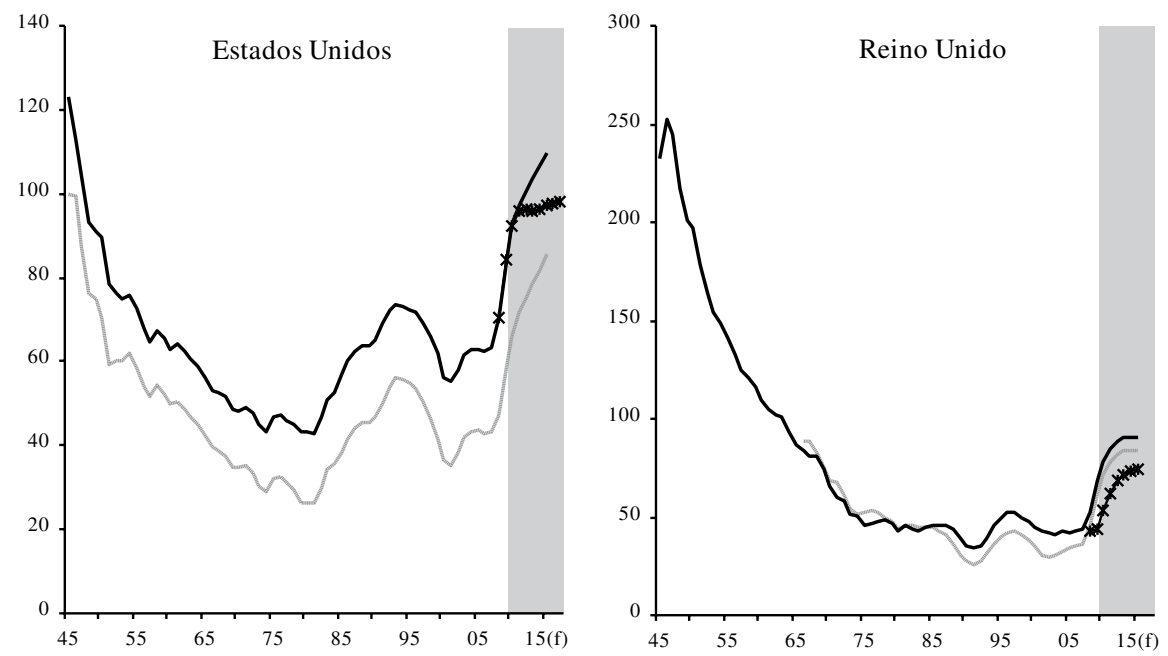

(f) Proyección del WEO (World Economic Outlook) de abril de 2010.

(1) En línea gruesa, deuda bruta; en línea delgada, deuda neta; la línea con asteriscos es la proyección oficial del CBO en EE.UU. y HM Treasury en el Reino Unido.

(2) Deuda del gobierno consolidado en Estados Unidos y del sector público en Reino Unido.

Fuente: Autoridad Nacional de Estadísticas del Reino Unido, Reserva Federal, Oficina de Análisis Económico de Estados Unidos y FMI.

continuarían siendo elevados (cercanos al 7\%). Por otra parte, la acumulación de deuda neta superaría el $80 \%$ alrededor del año 2012, lo que contrasta con el nivel que habían presentado ambas economías durante los últimos 30 años (ver Gráfico 4) ${ }^{1}$.

\section{EFECTOS MACROECONOMICOS DE LA EXPANSION FISCAL}

\subsection{Multiplicadores Fiscales}

En la literatura no ha habido un gran acuerdo respecto a la efectividad de los diferentes tipos de medidas fiscales temporales para lograr estimular la demanda agregada. Freedman et al. (2009) resume la evidencia respecto a los multiplicadores fiscales en

1 En ambas economías, la deuda externa pública alcanza a alrededor de $30 \%$ de la deuda pública bruta en 2009. 
el corto y largo plazo, junto con proveer estimaciones en base a esta discusión. Ilzetzki et al. (2009) muestran empíricamente que los multiplicadores fiscales en economías abiertas y con tipo de cambio flexible, son casi cero; a la vez, estos serían mayores en las economías desarrolladas que en las en vías de desarrollo, y considerablemente más persistentes. Pero también indican que un alto nivel de deuda pública a PIB, la duración y persistencia del efecto multiplicador disminuyen.

Los multiplicadores fiscales dependen, entre varias cosas, de cuánto se filtra del impulso fiscal en ahorro e importaciones, y en la respuesta de la política monetaria a las acciones fiscales, lo que puede variar de manera importante de una economía a otra. Así, economías más abiertas deberían tener un multiplicador menor (la expansión fiscal se "filtra" hacia las importaciones) que las economías cerradas. Una política monetaria coordinada con la política fiscal resulta en un multiplicador mayor, por cuanto se puede financiar el estímulo fiscal sin aumentar demasiado las tasas de interés reales.

Por otra parte, los canales de transmisión de las políticas fiscales varían según el tipo de política adoptada. Un aumento del consumo de gobierno tiene efectos adicionales: un mayor producto genera mayor ingreso, apoyando consumo adicional; un aumento de la inversión tiene efectos por el lado de la oferta, aumentando la productividad de los factores privados de producción, incrementando la respuesta del producto y disminuyendo la de la inflación ante el estímulo fiscal. Los recortes de impuestos y transferencias generan efectos por el lado de los ingresos y la riqueza.

Dentro de todas estas medidas, el efecto multiplicador es mayor en el consumo e inversión de gobierno que en los recortes de impuestos y transferencias, dado que estos últimos no tienen efectos directos en la demanda por bienes y servicios, además de depender de la propensión a gastar del ingreso o la riqueza ${ }^{2}$.

Freedman et al. (2009) también señalan que el efecto multiplicador del estímulo fiscal sería mayor si este fuese de mayor magnitud y apoyado por una política monetaria acomodaticia, si incluyera, además, medidas de apoyo a los sectores financieros en problemas. En tanto, estima que cada dólar gastado en inversión pública aumentaría el PIB en tres dólares; mientras las transferencias podrían hacerlo en un dólar.

\subsection{Sostenibilidad Fiscal}

No obstante lo anterior, un factor importante que podría afectar el valor del multiplicador fiscal es el stock de deuda pública del país, especialmente si los agentes económicos deciden ahorrar gran parte del estímulo fiscal ${ }^{3}$. Para que el estímulo sea exitoso es importante que no deteriore la sostenibilidad fiscal en el mediano plazo. Existe el riesgo de que los agentes económicos perciban que los grandes déficits fiscales incurridos en la actual coyuntura puedan ser difíciles de reducir a través del tiempo y puedan resultar en menor ahorro futuro y mayores tasas de interés reales, reduciendo

\footnotetext{
Dentro de ese mismo artículo se muestra evidencia al respecto.

Vale resaltar que las economías de países avanzados tienen mayor participación de agentes "ricardianos", una vez que tienen sistemas financieros más desarrollados permitiendo la intermediación financiera, necesaria para optimizar el perfil de consumo intertemporal de los agentes.
} 

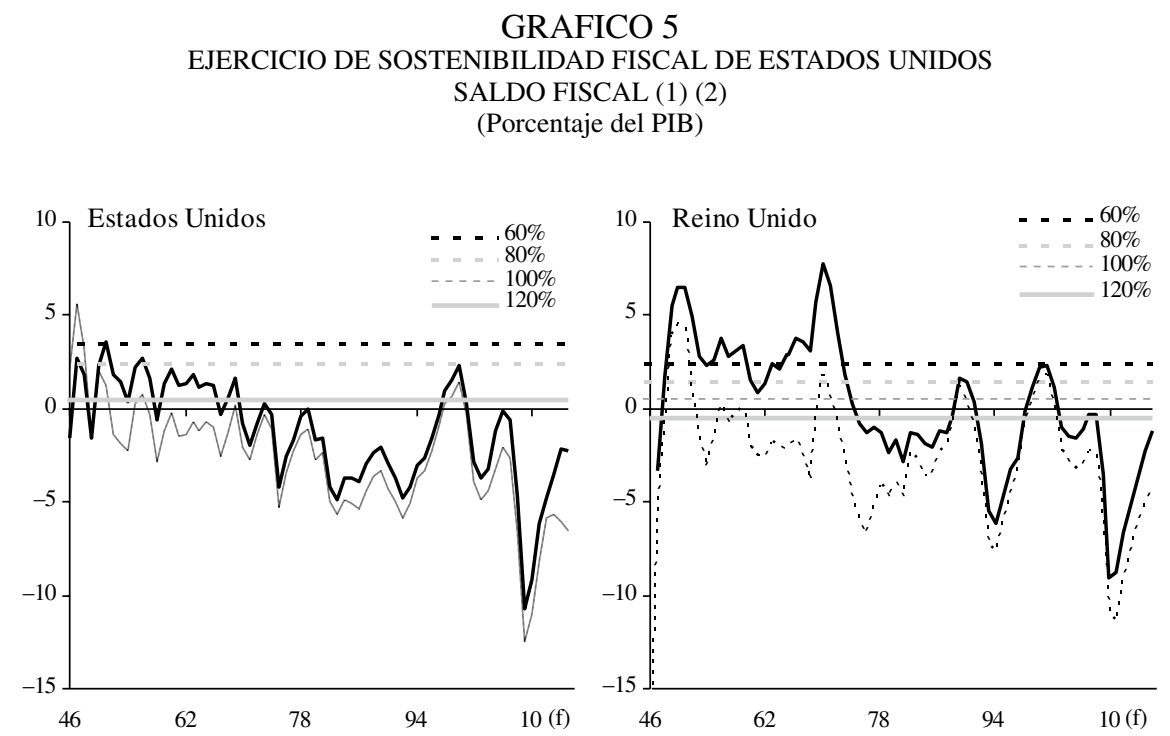

(1) Saldo Fiscal del Gobierno Consolidado (primario en línea sólida y financiero, punteada). Las líneas horizontales representan el déficit anual requerido para reducir la deuda fiscal a PIB al porcentaje indicado, entre 2014 y 2029.

(2) Proyecciones de WEO de abril de 2010.

Fuentes: Bureau of Economic Analysis, FMI, Mitchell (2007), UK National Statistics Authority y Her Majesty's Treasury.

la inversión y la capacidad productiva. Por lo tanto, el tema de la sostenibilidad fiscal resulta de gran importancia.

De acuerdo al análisis de FMI (2009a y 2010), basado en un marco conceptual simple de evaluación de estabilidad de deuda, descrito en detalle en el anexo, los esfuerzos necesarios para estabilizar la deuda pública a un nivel considerado sostenible serían de magnitud considerable. Economías como Estados Unidos y Reino Unido deberían lograr superávits promedio de 4,3\% y 3,4\% del PIB, respectivamente, para lograr reducir la deuda desde los niveles proyectados de $112,0 \%$ y $99,7 \%$ en 2014 , a un $60 \%$ en 2029. Este es un nivel muy alto en comparación con los niveles históricos (Gráfico 5).

Utilizando los datos de deuda pública bruta y superávit primario utilizados en su análisis, más el crecimiento del PIB a precios corrientes del WEO de octubre de 2009 y un supuesto de un diferencial de $1 \%$ a favor de la tasa de interés nominal de largo plazo, realizamos el ejercicio para diferentes niveles de deuda objetivos. Para Estados Unidos, los superávits fiscales registrados posterior a la Segunda Guerra Mundial y a finales de los años noventa permitirían estabilizar la deuda a niveles de entre $80 \%$ y $100 \%$. En el caso de Reino Unido, esto sería posible con el esfuerzo fiscal realizado 
al principio de la década de los 90 y 2000. Sin embargo, las proyecciones oficiales en Estados Unidos y Reino Unido de déficit fiscal arrojan saldos fiscales por debajo de esos niveles ${ }^{4}$.

Otro punto importante de destacar es que las estimaciones de FMI (2009a y 2010) consideran un diferencial de tasas de interés y crecimiento de $1 \%$. El CBO, en su presupuesto de enero de 2010, estima una tasa de interés de largo plazo de 5,5\% en 2019 y un crecimiento del PIB nominal de 4,2\%, arrojando un diferencial de 1,7\%. Utilizando este supuesto más conservador ${ }^{5}$, los requerimientos de superávit primario serían aún mayores.

En síntesis, dado lo descrito anteriormente, se estima que las economías de Estados Unidos y Reino Unido presentarían niveles de deuda en relación a PIB cercanos a $100 \%$. Estos altos niveles se estabilizarían si estas economías hicieran ajustes fiscales de contención del déficit en línea con ajustes observados históricamente.

\subsection{Canales de Transmisión de Déficits Fiscales hacia las Tasas de Interés}

Aunque los altos déficits y el aumento de la deuda pública en las principales economías avanzadas no han impactado fuertemente las tasas de interés, hasta el momento, esto se ha debido, en parte, a una retracción del consumo, producto en gran medida de un sobreendeudamiento de los hogares, mayores entradas de capitales y una política monetaria extraordinariamente expansiva, que incluyó fuertes inyecciones de liquidez a través del credit easing. Una vez que los estímulos sean retirados y que los balances de los hogares se equilibren, los altos déficits fiscales podrían provocar alzas en las tasas de interés de largo plazo en estas economías.

Algunos de los canales por los cuales el alza del déficit fiscal provoca aumentos de tasas de interés de largo plazo son: (i) la reducción del ahorro nacional, a través del menor ahorro fiscal, (ii) el aumento de la prima por riesgo fiscal (en función del monto acumulado de deuda pública), (iii) el riesgo de roll-over de deuda una vez que los plazos se acortan y (iv) el aumento de las expectativas de inflación.

Respecto del canal del ahorro, lo que se ha dado es un aumento neto del ahorro privado que ha compensado el desahorro neto fiscal (además de entradas de capital en la mayoría de los países, en general, por la compra de instrumentos libres de riesgos emitidos en estos mercados) (Gráfico 6). El aumento del ahorro privado observado en 2009 está asociado, en parte, a un fuerte desapalancamiento de los hogares en curso en varias economías, entre ellas Estados Unidos. Además, las expectativas de los agentes acerca de la necesidad de un esfuerzo fiscal futuro que implique más altos impuestos también tienen un rol importante en sus decisiones de ahorro (equivalencia ricardiana). Como resultado, un mayor ahorro privado, combinado con el ahorro externo de países con superávits en cuenta corriente, resulta en cantidades altas de

4 En el caso de considerar un horizonte más largo, de alrededor de 30 años, los déficits mencionados permitirían reducir los niveles de deuda hasta un 60\%. Otro punto importante es que, en la coyuntura actual, las bajas perspectivas para el crecimiento y la inflación ponen una dificultad adicional para las medidas de estabilización fiscal.

5 El CBO, no obstante, tiene un perfil de déficits más bajo que el FMI. 


\section{GRAFICO 6}

SALDOS FINANCIEROS

(Porcentaje del PIB)
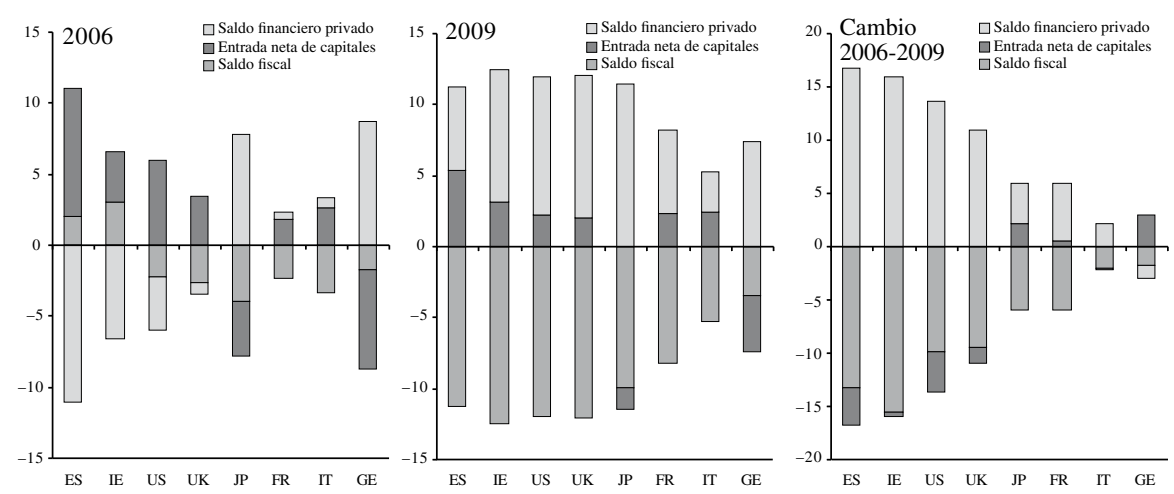

Fuente: Moody's.

ahorro corriente, lo que limita el alza de las tasas de interés de largo plazo. Otros factores estructurales que favorecen la decisión de ahorro doméstico y externo y, por lo tanto, inciden en el bajo nivel de tasa de interés en curso en economías avanzadas se deben a la mayor apertura de capitales y mercados de deuda más profundos en estas economías (Aisen y Hauner, 2008). La reversión de algunos de estos elementos que pueden ser transitorios elevaría las tasas de interés en futuro respecto a los niveles observados en 2009.

El segundo canal, la percepción de riesgos, se ha mantenido relativamente moderada. En lo más reciente, los problemas de deuda de las economías de la Eurozona periférica (Portugal, Italia, Irlanda, Grecia y España) han traído el foco de vuelta hacia la situación fiscal (ver Aisen et al., 2010). A pesar de esto, los spreads del CDS a cinco años, de Reino Unido y Estados Unidos, siguen siendo bajos (ver Gráfico 7). Es importante mencionar que la alta demanda por los instrumentos de renta fija de EE.UU., como resultado de la aversión al riesgo, se ha visto reforzada por las inyecciones de liquidez y, en algunos casos, con compras por parte de Bancos Centrales, como el caso de la Reserva Federal. Sus compras netas alcanzaron a alrededor de US\$ 300 miles de millones durante 2009, representando el $21 \%$ de las emisiones netas realizadas por el Tesoro durante ese período. En el Reino Unido, por otra parte, el plan original era comprar hasta $£ 100$ miles de millones (7\% del PIB). Al 28 de enero de 2010, había comprado gilts por un monto muy superior, cercano a £198 miles de millones.

En cuanto al riesgo de roll-over, en el caso de Estados Unidos, el porcentaje de deuda que madurará en un año aumentó considerablemente, alcanzando a US\$2.670 miles de millones a agosto de 2009, para reducirse lentamente a US\$2.490 en marzo 


\section{GRAFICO 7}

CDS SOBERANO

(Puntos base)

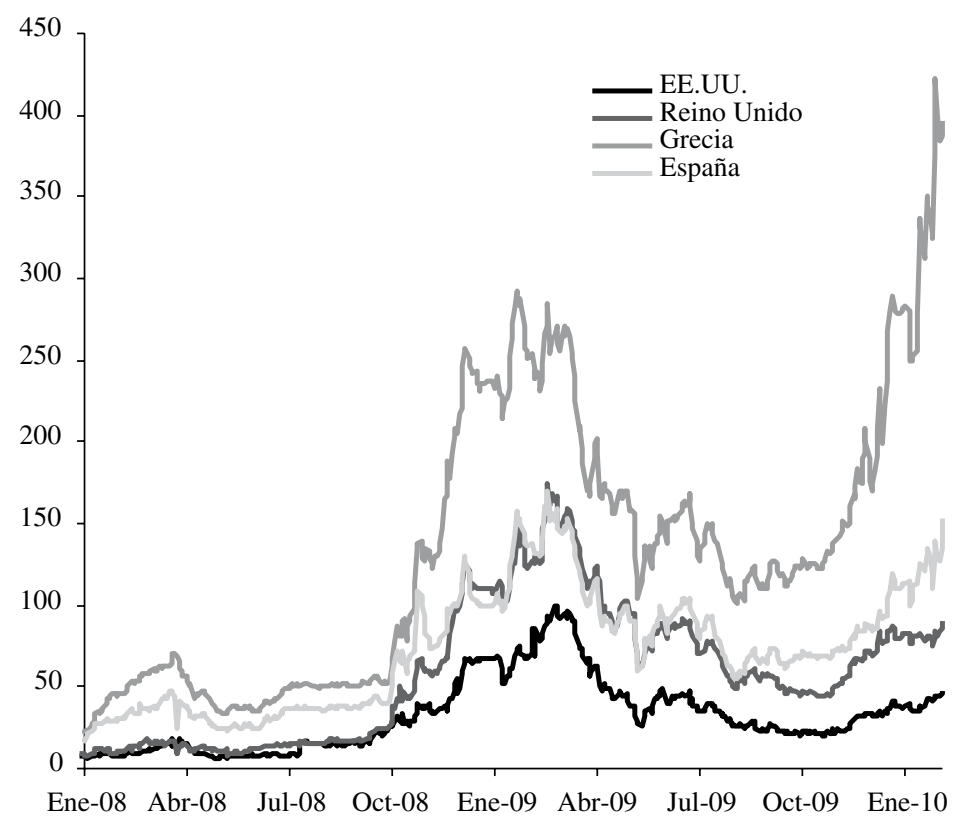

Fuente: Thomson Datastream.

de 2010. Los plazos llegaron a 3,8 años en promedio durante octubre de 2008, lo que, sin embargo, se había observado en aún menores magnitudes durante los años 70 (Gráfico 8). A pesar de esto, el Tesoro ha aumentado la participación de bonos de más largo plazo en las recientes emisiones.

Las expectativas de inflación a largo plazo, por su parte, se han mantenido relativamente estables, según el consenso de mercado. Esto se asocia a una visión de una recuperación lenta de la economía mundial y de presiones inflacionarias controladas (FMI, 2009e).

\subsection{Estimaciones y Efecto Desplazamiento sobre las Economías Emergentes}

El efecto desplazamiento, o crowding-out, que se produce generalmente en crisis financieras internacionales como en 1929, principios de los años ochenta o en la reciente crisis subprime, puede tomar distintas formas, como el mayor cobro de intereses sobre la deuda (aumento de los spreads cobrados), el otorgamiento de me- 


\section{GRAFICO 8}

MADUREZ PROMEDIO DE LA DEUDA DEL TESORO DE EE.UU. Y PORCENTAJE QUE MADURA DENTRO DE UN AÑO

(Promedio anual, porcentaje)

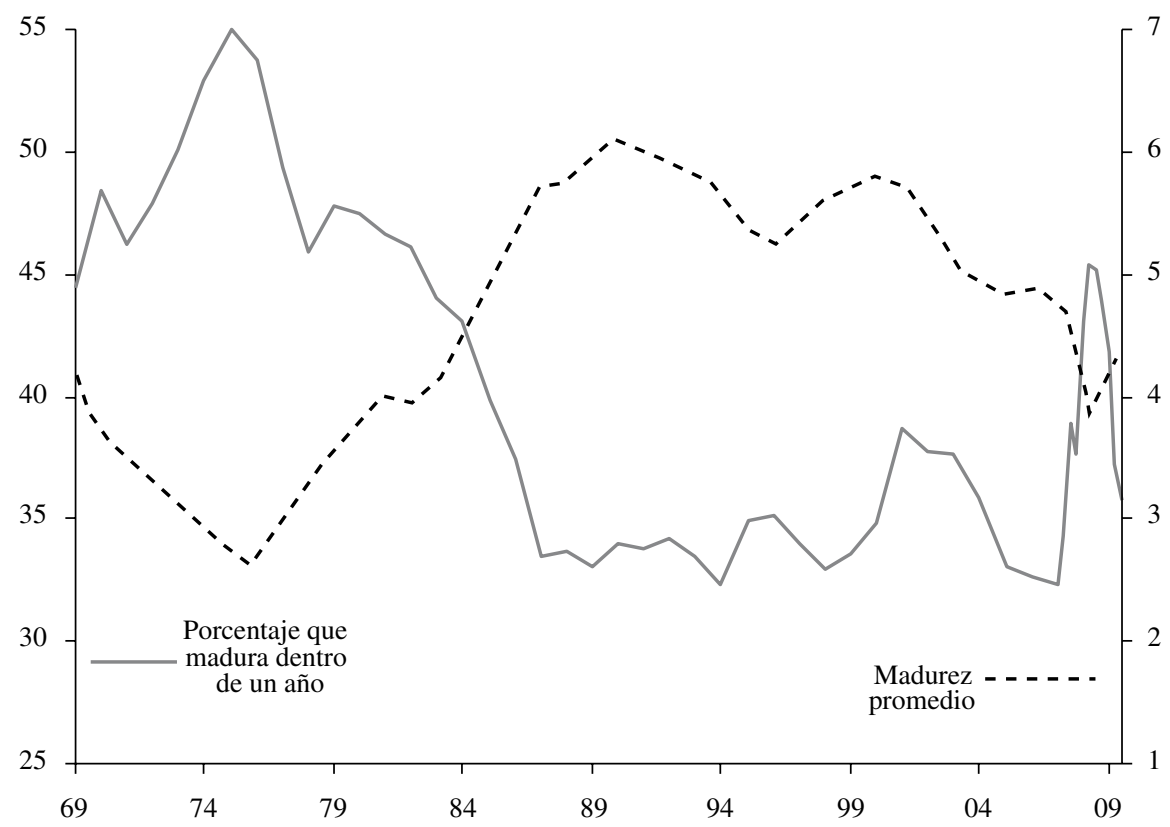

Nota: Ultimo dato, corresponde al mes de marzo de 2010.

Fuente: Monthly Treasury Statement, varias ediciones.

nores montos de préstamos o menores plazos y, en algunos casos, incluso el cierre de las líneas de crédito en el exterior o la imposibilidad de colocar bonos en el mercado internacional. Durante la Gran Depresión (Reinhart y Reinhart, 2009), las economías emergentes no tuvieron acceso a mayor endeudamiento como sí lo tuvieron las economías más avanzadas, mientras que en el segundo caso, el fuerte aumento de la tasa de política monetaria durante la era Volcker trajo consigo la Crisis de la Deuda. En este episodio, el impacto negativo del alza de tasas de intereses en Estados Unidos sobre el servicio de la deuda de países en América Latina produjo dudas sobre la sostenibilidad fiscal y externa provocando una fuerte huida de capitales de la región. Esta salida de capitales solo sería atenuada posteriormente con la reestructuración de la deuda propuesta por el Plan Brady.

Durante la reciente crisis subprime, sin embargo, las economías emergentes se encontraban en un pie diferente. Varias se encontraban en proceso de reducción de su deuda externa, de mayor emisión en sus mercados locales y mayor crecimiento económico (Cowan et al., 2006). Luego de la caída de Lehman Brothers, los spreads 


\section{GRAFICO 9}

\section{TASA DE INTERES DEL EMBI GLOBAL, TASA DE INTERES DE LA DEUDA SOBERANA DE CHILE Y TASAS DE LARGO PLAZO DE EE.UU. \\ (Porcentaje)}

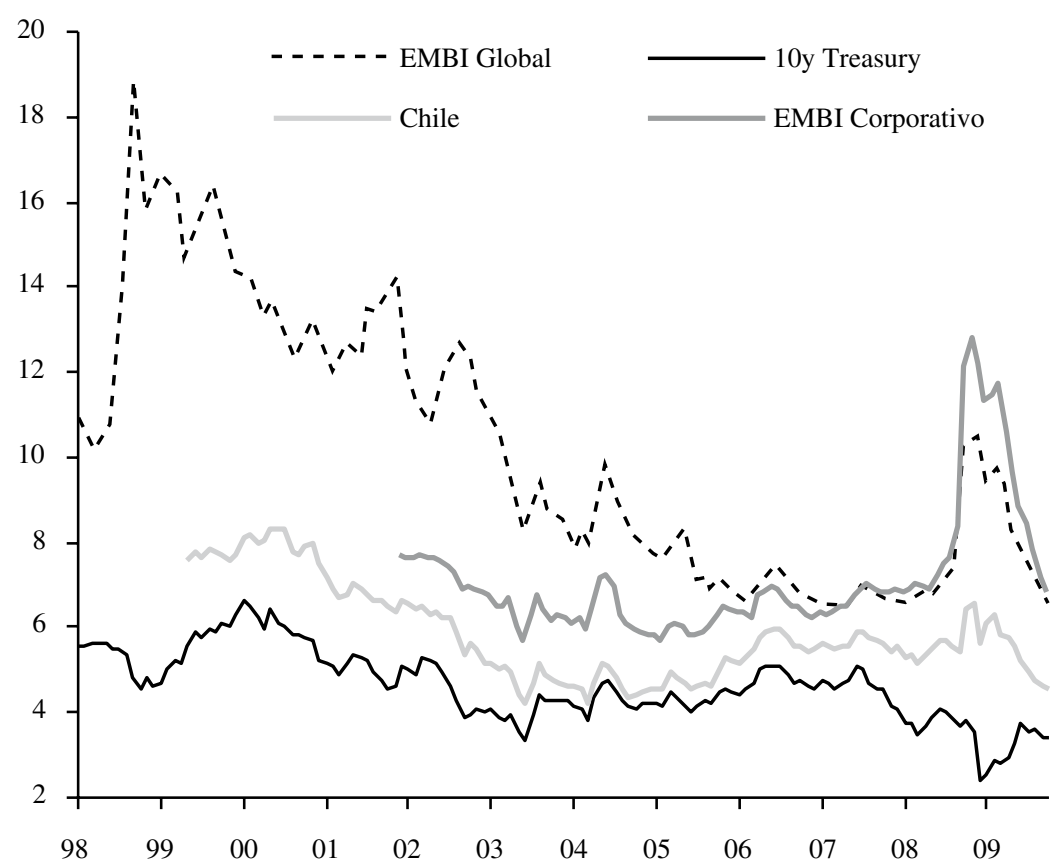

Fuente: Bloomberg.

de las economías emergentes aumentaron, aunque a un nivel promedio menor que en 1998, para recuperarse rápidamente en los meses posteriores (ver Gráfico 9).

De lo expuesto anteriormente, surge la importancia de estimar los efectos de los mayores déficits y deuda pública en las economías avanzadas, especialmente EE.UU., en sus tasas de interés de largo plazo y, consecuentemente, en los spreads de la deuda de economías emergentes.

La relación entre los mayores déficits fiscales sobre la tasa de interés se puede apreciar en el Gráfico 10. De una manera simple, esto ilustra el efecto de un punto adicional de déficit fiscal a PIB, que elevaría las tasas en alrededor de 30 y 40 puntos base para Estados Unidos y Reino Unido, respectivamente. Sin embargo, desde 2000 en adelante, esta relación se ha quebrado, en parte, por mayores compras de bonos del Tesoro, en parte por la posible escasez de activos seguros (Caballero, Farhi y Gourinchas, 2008). 


\section{GRAFICO 10}

\section{RELACION ENTRE LAS TASAS DE INTERES Y LOS DEFICITS FISCALES \\ (Porcentaje)}
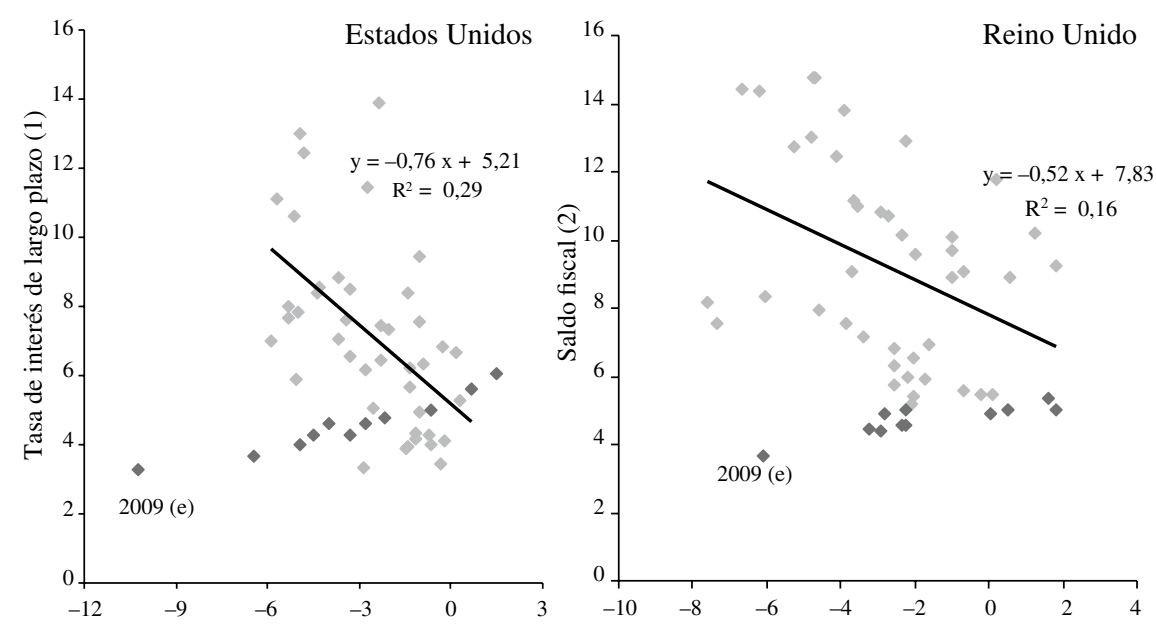

(e) Estimación. Línea de regresión abarca la información entre 1957 y 1999 (puntos grises).

(1) Tasa de interés del bono nominal de Gobierno de 10 años plazo.

(2) Saldo financiero, porcentaje del PIB.

Fuentes: Bloomberg, BEA y UK National Statistics.

En la Tabla 1 se realiza una estimación basada en Laubach (2009) considerando las expectativas de déficit fiscal y deuda pública a cinco años (en porcentaje del PIB), las de inflación a cinco años y de la TPM a un año para Estados Unidos. Adicionalmente, se consideran el ahorro nacional total y las compras de bonos (en porcentaje del PIB) en línea con los canales de transmisión mencionados anteriormente. En la columna 1 se muestra que, a simple vista, un aumento de 1 punto porcentual (pp.) del déficit lleva a un aumento de 37 punto base (pb.) en la tasa de interés de largo plazo; este efecto no sería significativo si se incluyen otras variables. Sin embargo, al utilizar una medida de tasa de interés real de largo plazo (la tasa nominal menos la expectativa de inflación a 10 años), implicaría que un aumento de $1 \mathrm{pp}$. llevaría a un aumento de entre 11 y 12 puntos base en la tasa de interés real. Suponiendo que se mantienen las compras de bonos del Tesoro por los extranjeros y la tasa de ahorro actual de la economía (al primer trimestre de 2010), si utilizamos las proyecciones del WEO de abril de 2010 con déficit fiscal de 6,5\% en 2015, una inflación de 2,2\% y la TPM en $4 \%$ llevarían a la tasa a casi 5,0\%, implicando un aumento de 160 puntos base, lo que está en línea con otras estimaciones. En estimaciones usando datos en panel y 


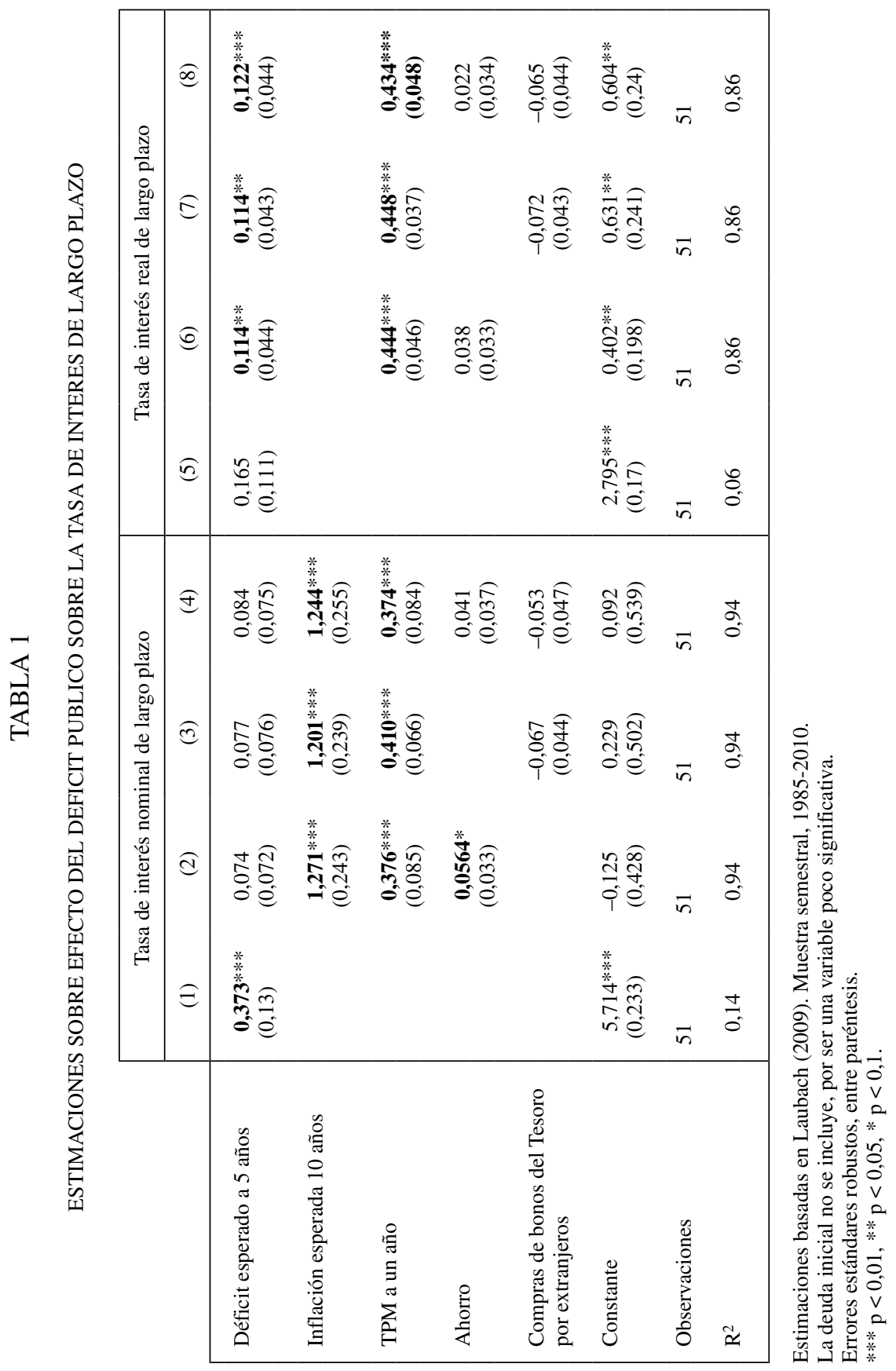


que consideran más variables, se encuentran efectos significativos que alcanzarían a entre 10 y 60 puntos base (IMF, 2009c), y que pueden ser mayores considerando los déficits fiscales esperados (Laubach, 2009) ${ }^{6}$.

El aumento de la tasa de interés de largo plazo en EE.UU. (y en general de las economías avanzadas) genera preocupación respecto a la capacidad de las economías emergentes de financiarse externamente, dado el aumento del spread de tasas de bonos emergente sobre las del Tesoro americano que esto implicaba (Gráfico 11). Los canales mediante los cuales esto ocurre, no parecen ser demasiado evidentes. Por una parte, un incremento en las tasas de los bonos del Tesoro en EE.UU., un activo con muy poco riesgo de default, puede estar asociado a un aumento en los rendimientos de todos los activos de mayor riesgo, incluida la deuda de economías emergentes, debido a que los inversionistas buscan mayor compensación por el riesgo extra. A su vez, la percepción de riesgo soberano de estas economías puede aumentar, debido a mayores servicios de la deuda y, por lo tanto, el spread aumentaría.

Las estimaciones realizadas en FMI (2009d) para el período 1997-2006 implican que un aumento de la deuda pública de EE.UU. en 1 pp. resulta en spreads mayores en $0,4 \%$, agregándose también el efecto del aumento de $0,1 \%$ por cada punto base que aumenta la tasa larga. Con esto, si se considera un aumento de 40 puntos porcentuales de la deuda pública sobre PIB, los spreads pueden llegar a aumentar en $16 \%$, sin considerar los efectos adicionales del aumento de la tasa larga, que de aumentar 160 pb. agregaría otro $16 \%$ adicional. Si consideramos un spread como el de Chile, de 120 pb., este puede llegar a casi 160 pb. ${ }^{7}$.

Sin embargo, un punto importante que debe hacerse es respecto al riesgo implícito que conlleva esta mayor deuda pública en Estados Unidos. En Krishnamurthy y Vissing-Jørgenssen (2007) se muestra evidencia de que existe una correlación negativa entre la deuda federal de EE.UU., como porcentaje del PIB, y el spread entre los bonos corporativos y los del Tesoro. Se argumenta que esta relación refleja un convenience yield en la deuda pública, que disminuye su valoración marginal a medida que aumenta el stock de deuda. Esto es atribuible a la superior liquidez y bajo riesgo de incumplimiento en el pago. Es posible que esta relación también aplique al caso de los bonos de economías emergentes, a menudo considerados como una clase de activo similar a los bonos corporativos de alto rendimiento de Estados Unidos. Después de la caída de Lehman Brothers, las tasas de interés de los bonos del Tesoro bajaron, mientras los spreads de las economías emergentes se incrementaron. Esto puede constituir evidencia a favor de este punto, aunque no tiende a darse en el largo plazo, como se muestra en FMI (2009d).

Otra situación, que no puede ser descartada, es la posibilidad de que el aumento en los spreads no solo sea menor, sino que pueda disminuir, aun cuando las tasas de

6 Cabe destacar que el CBO, en enero de 2010, proyectó una tasa de interés de largo plazo de 5,5\%, con un déficit para 2015 sustancialmente menor al del WEO (2,6\% del PIB). Otro punto, es que las estimaciones de IMF (2009c) no consideran el cambio en la tasa de política monetaria.

7 Este cálculo no considera cambios en otras variables que controlan las estimaciones de los estudios mencionados anteriormente, como proyecciones de crecimiento de EE.UU. y de cada economía, el $V I X$, entre otros. 


\section{GRAFICO 11}

SPREAD EMBI GLOBAL Y TASAS DE LARGO PLAZO DE EE.UU.

(Puntos base, porcentaje)

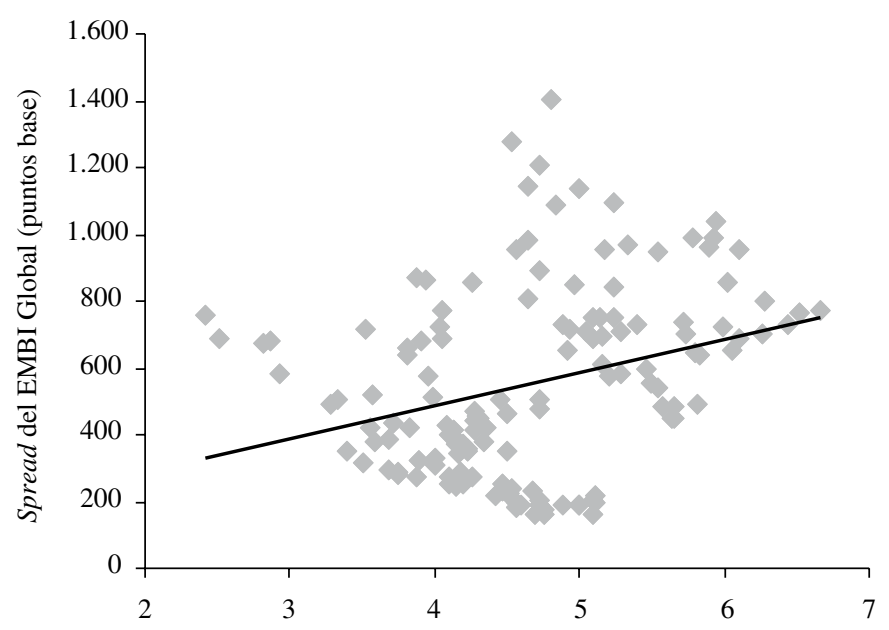

Tasa de interés nominal a 10 años de bonos del Tesoro de EE.UU. (porcentaje)

Fuente: Bloomberg.

interés de largo plazo aumenten en las economías avanzadas, especialmente en el caso de economías emergentes cuya deuda sea percibida como menos riesgosa. Algo de esto se vio durante la reciente crisis fiscal en Europa, donde los spreads de las economías periféricas (España, Grecia, Irlanda, Italia y Portugal) aumentaron en mayor grado que en las economías emergentes (Banco Central de Chile, 2010).

\section{CONCLUSION}

El objetivo de este artículo fue analizar el impacto del aumento de los déficits y deuda públicos en los países avanzados producto de la expansión fiscal contracíclica sin precedentes implementada por estos países durante la crisis financiera del 2008.

La evaluación de los riesgos macroeconómicos para estas economías indica que el principal riesgo sería que esta expansión fiscal provocara un efecto desplazamiento del consumo e inversión privados que pusiera en riesgo el crecimiento del PIB en el futuro. Este efecto desplazamiento sería resultado del aumento de las tasas de interés de la deuda en Estados Unidos y podría también ser relevante para la deuda soberana y corporativa de países emergentes. 
Si bien la evidencia histórica sugiere que alzas de tasas de interés en Estados Unidos están relacionadas a mayores niveles de spreads soberanos, la coyuntura actual muestra algunas importantes diferencias. Muchos países emergentes, Chile entre ellos, adoptaron un marco macroeconómico más equilibrado y redujeron sus deudas públicas como porcentaje del PIB, fortaleciendo su posición fiscal de manera significativa. Si bien es cierto que lo anterior debería atenuar el efecto de alzas de tasas de interés en los países avanzados, persiste el riesgo de que no sean tan bien absorbidas, dificultando el acceso a financiamiento externo en estos países.

\section{BIBLIOGRAFIA}

AISEN, A., I. BADILLA, F. JAQUE y D. MORENO (2010). “Crisis en la Zona Euro: Fundamentos tras el Reciente Aumento de Spreads Soberanos", Revista de Economía Chilena 13 (1), pp. 83-93.

AISEN, A. y D. HAUNER (2008). "Budget Deficits and Interest Rates: A Fresh Perspective", Working Paper 08/42, Fondo Monetario Internacional.

BANCO CENTRAL DE CHILE (2010). Informe de Estabilidad Financiera, Primer Semestre.

CABALLERO, R., E. FARHI y P. O. GOURINCHAS (2008). "Financial Crash, Commodity Prices and Global Imbalances", Brooking Papers on Economic Activity, Fall, pp. 1-55.

COWAN, K., E. LEVI-YEYATI, U. PANIZZA y F. STURZENEGGER (2006). "Public Debt in the Americas: New Data and Stylized Facts", Documento de Trabajo. Junio.

FONDO MONETARIO INTERNACIONAL (2009a). The State of Public Finances Cross-Country Fiscal Monitor, IMF Staff Position Note, Julio.

FONDO MONETARIO INTERNACIONAL (2009b). The State of Public Finances Cross-Country Fiscal Monitor, IMF Staff Position Note, Noviembre.

FONDO MONETARIO INTERNACIONAL (2009c). Global Financial Stability Report, Octubre.

FONDO MONETARIO INTERNACIONAL (2009d). United States: Selected Issues, Country Report 09/229.

FONDO MONETARIO INTERNACIONAL (2009e). World Economic Outlook, Octubre.

FREEDMAN, C., M. KUMHOF, D. LAXTON y J. LEE (2009). "The Case for Global Fiscal Stimulus", IMF Staff Position Note 09/03.

GALE, W. y P. ORSZAG (2003). "Economic Effects of Sustained Budget Deficits", National Tax Journal 56 (3), pp. 463-485.

HORTON, M. y A. IVANOVA (2009). "The Size of the Fiscal Expansion: An Analysis for the Largest Countries", Fondo Monetario Internacional, Departamento de Asuntos Fiscales.

ILZETZKI, E., E. MENDOZA y C. VEGH (2009). "How big (small?) are fiscal multipliers?", Documento preliminar. Septiembre.

KRISHNAMURTHY, A. y A. VISSING-JØRGENSSEN (2007). “The Demand for Treasury Debt”, NBER Working Paper Nr.12881, National Bureau of Economic Research.

LAUBACH, T. (2009). "New Evidence on the Interest Rate Effects of Budget Deficits and Debt", Journal of the European Economic Association 7 (4), pp. 858-885.

MITCHELL, B. R. (2007). International History Statistics, Europe 1750-2005. Palgrave Macmillan, Londres. REINHART, V. y C. REINHART (2009). Is there scope for fiscal stimulus for debt-intolerant countries? VOX, 22 August. http://www.voxeu.org/index.php?q=node/3893. 


\section{ANEXO \\ SOSTENIBILIDAD FISCAL}

Una manera simple de hacer la evaluación de sostenibilidad fiscal es partir de la ecuación de la evolución de la deuda pública, donde el stock de deuda en $t, B_{t}$ es igual al stock que había al principio del período con los intereses y menos (más) el superávit (déficit) primario generado durante el período ${ }^{8}$ :

$$
B_{t}=(1+i) B_{t-1}-S P_{t}
$$

Normalizando en porcentaje del PIB, tenemos:

$$
b_{t}=\frac{(1+i)}{(1+\lambda)} b_{t-1}-s p_{t}
$$

Donde $b_{t}$ corresponde a la deuda pública bruta como porcentaje del PIB, $i$ a la tasa de interés nominal, $\lambda$ a la tasa de crecimiento del PIB a precios corrientes (que se suponen en sus niveles de largo plazo) y $s p_{t}$ al superávit primario en el período $t$. Para que la deuda se estabilice, el superávit primario necesario para sostener la deuda en un nivel constante sería de:

$$
s p^{*}=\frac{(i-\lambda)}{(1+\lambda)} b_{t}
$$

En la actual coyuntura, sin embargo, lo que se desea es reducir el endeudamiento público a un determinado nivel, por ejemplo, $b^{*}$, en un plazo de $n$ períodos. Para simplificar el cálculo, establecemos que $\theta=(1+i) /(1+\lambda)$. Con esto, el superávit promedio requerido para llevar a cabo esta reducción sería de:

$$
\begin{aligned}
b_{t+n} & =b^{*}=\theta b_{t+n-1}-s p_{t+n}=\theta^{2} b_{t+n-2}-\theta s p_{t+n-1}-s p_{t+n} \\
\ldots & =\theta^{n} b_{t}-\sum_{j=0}^{n} \theta^{j} s p_{t+n-j}=\theta^{n} b_{t}-s p^{*} \sum_{j=0}^{n} \theta^{j} \\
\ldots & =\theta^{n} b_{t}-s p * \frac{1-\theta^{n}}{1-\theta}
\end{aligned}
$$

8 En el ejercicio no se incluyen las rentas generadas por el señoreaje. 


$$
s p^{*}=\left(\frac{1-\theta}{1-\theta^{n}}\right)\left(\theta^{n} b_{t}-b^{*}\right)
$$

En el caso de que $i>\lambda$ (entonces $\theta>1$ ), se requerirá un superávit primario para estabilizar la deuda. El superávit requerido, por supuesto, también aumentará mientras menor sea el plazo establecido para alcanzar la meta. 
